\title{
Alternateurs de 65.000 KVA de la Niagara Falls Power Company
}

Depuis la fin du XIXe siècle, époque à laqquelle commença l'utilisation de l'immense ressource d'énergie que constituent les chutes du Niagara, de très grands progrès furent réalisés pour tirer le meilleur parti de cette richesse naturelle. Celle-ci est évaluée à quatre millions de chevaux pouvant être utilisés sans amoindrir la beaulé du site et le $1 / 6$ seulement a été capté. (Un trailé signé d'un commun accord par les Etals-Unis ct le Canada en règlemente d'ailleurs tous les projets).

L'augmentation croissante du prix du combustible et de son transport d'une part et l'intérêt de ménager les gisements de houille d'autre part ont conduit les grandes firmes américaines à équiper leurs centrales arec des unités de plus en plus puissantes, moins encombrantes et moins coûteuses à puissance égale. Presque tous les projets en cours de réalisation ou à l'étude comportent des puissances de 20 à $30.000 \mathrm{KW}$. sans se soucier

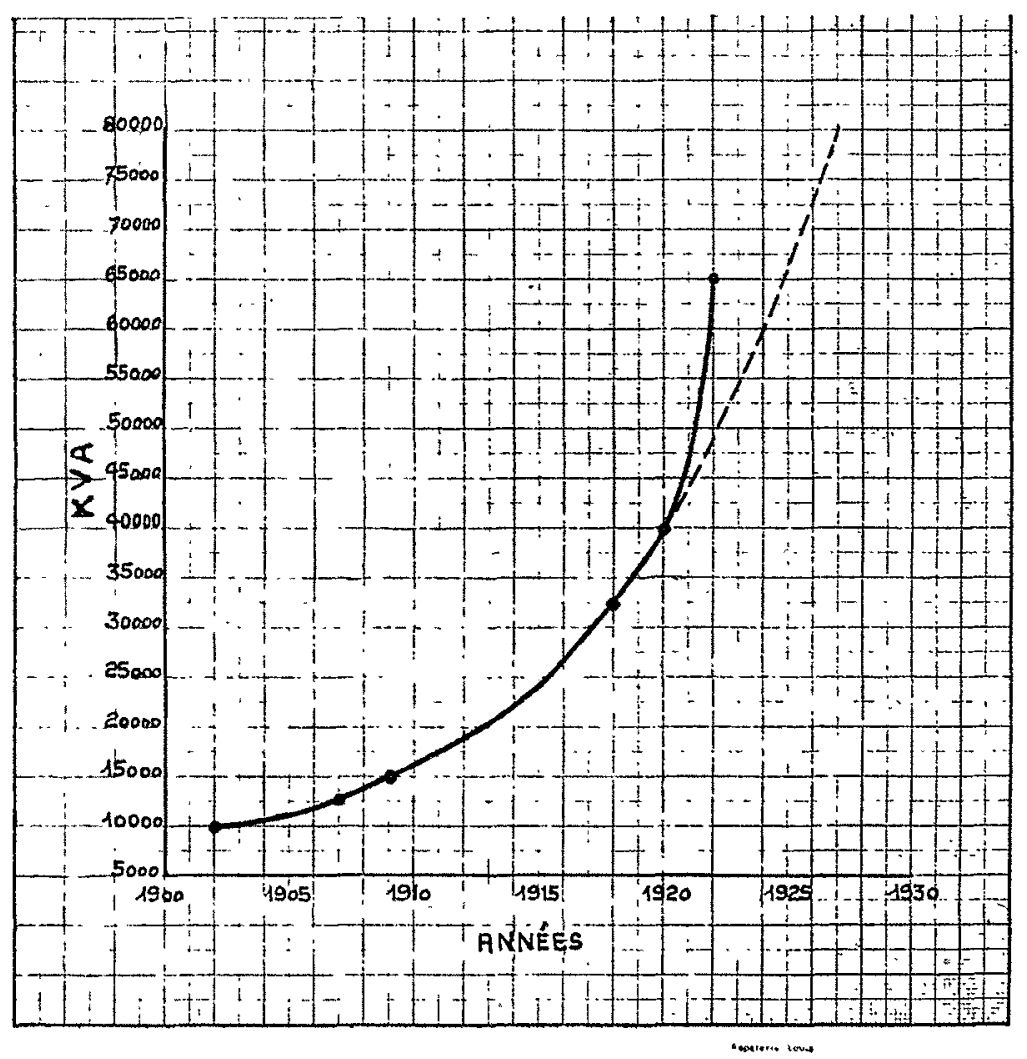

Fig. 1

de répartir en plusieurs groupes les puissances des stations du fait qu'un nombre suffisant de centrales en parallèle rend moins utiles les réserves et que la possibilité de régler pour chacune d'elles le débit au moyen de réservoir permet d'utiliser chaque groupe dans les conditions de charge les plus favorables, quel que soit le débit moyen.

Ainsi dans cette ancienne centrale où ont été installés à l'origine onze groupes de $3.750 \mathrm{KW}$. deux nouvelles unités de chacune $65.000 \mathrm{KVA}$ sont prévues et la première a été mise en service en décembre 1923 à la Niagara Falls Power et $C^{\circ}$ sur la rive américaine.

La fig. I donne une idée claire de l'augmentation progressive

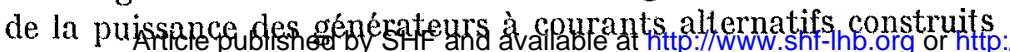

en particulier par la Général Electric Co durant ces vingt dernières années. Alors qu'en 1909 le plus grand alternateur a une puissance de l'ordre de $15.000 \mathrm{KVA}$, il atteint en $191832.500 \mathrm{KVA}$ en $192040.000 \mathrm{KVA}$, en 192265.000 . Ceci montre également que la puissance de ces machines a été doublée durant les quatre dernières années.

Le rendement global des groupes turbo-alternateurs a également fait des progrès sensibles et il est particulièrement intéressant de noter les rendements des groupes de $65.000 \mathrm{KW}$. dont la turbine développe 70.000 chevaux sous 65 mètres de chute.
$65.000 \mathrm{KVA}-$
$65.000 \mathrm{KVA}-$
$65.000 \mathrm{KVA}-$
- Cos 0,8 - Rendement $97,5 \%$
Cos 0,9 - Rendement 97,8
$\operatorname{Cos} \odot 1$ - Rendement 98,1

La fig. 2 donne les courbes de marche à vide et à pleine charge pour $\cos ?=1$ et $\cos ?=0,8$.

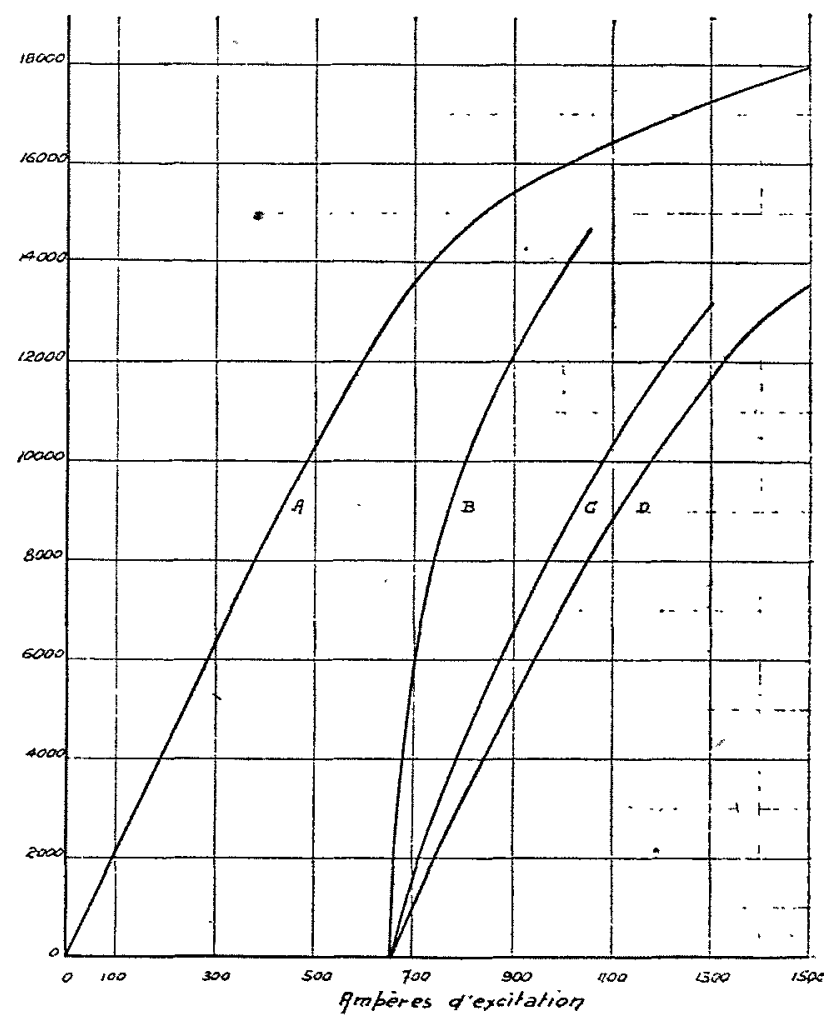

Fig. 2

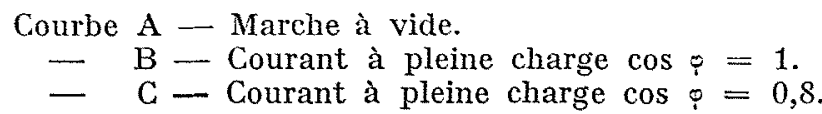

Les températures garanties étant les suivantes : Pour 65.000 KVA $\operatorname{Cos} \varphi$ 0,8 la surélévation de température du stator ne doit pas dépasser $50^{\circ} \mathrm{C}$. mesurẻe au thermomètre et $60^{\circ}$ de surélévation indiquée par les détecteurs de température. La garantie pour le rotor est de $55^{\circ} \mathrm{C}$., surélévation mesurée par variation de résistance. Les générateurs doivent pouvoir supporter une surcharge de $5 \%$ et une tension de 13.200 volts sans que l'isolement soit détérioré.

Les détecteurs de température, au nombre de 24 , sont placés dans le stat nuer indiquer la température du cuivre. Quelques- 
uns sont placés dans les encoches entre les sections clles-mèmes, les autres à la base des encoches entre la section du bobinage et les tôles de linduit.

La fig. 3 donne les caractéristiques suivantes:

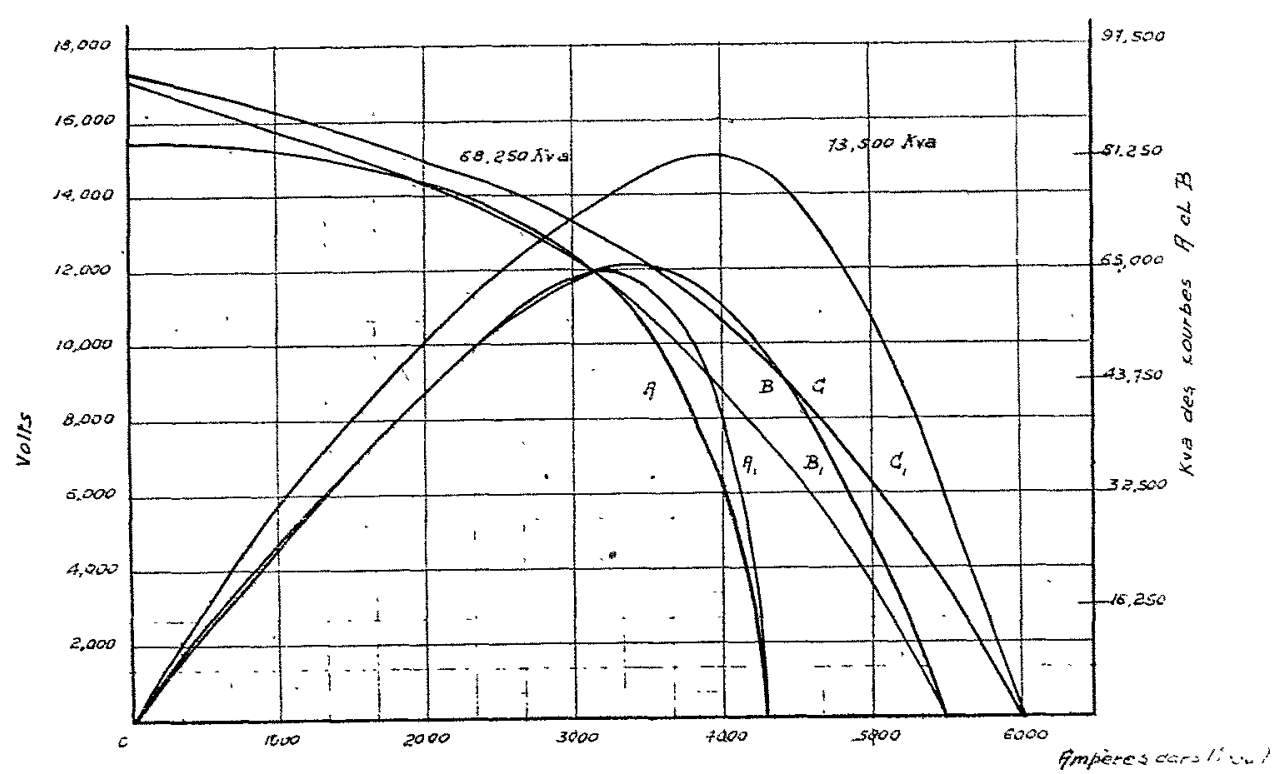

Fig. 3

Courbe $\mathrm{A}-\mathrm{A}_{1}-\cos \%=1 \quad 12.00 \mathrm{c}$ volts $65.000 / \mathrm{KVA}$

- $\mathrm{B}-\mathrm{B}_{1}-\cos \%=0,8 \quad 12.000$ volts $65.000: \mathrm{KVA}$

- $\quad \mathrm{C}_{-} \mathrm{C}_{1}-\cos \%=0,8 \quad 13.200$ volts $68.200, \mathrm{KVA}$ de leur turbine respective. Les denx dernières unilés installées peuvent ètre arrêtées rapidement en cas d'emballement de la turbine, tandis que les anciennes ne possédaient aucuu disposilif spécial de freinage.

Leur coefficient de poids n'est que $10 \mathrm{~kg} .5$ par KVA, tandis qu'il atteignait $23 \mathrm{~kg}$. par KVA dans les premières unités. Bien que ce poids paraisse réduit, il est relativement grand comparé à celui d'un alternateur de grande puissance à 60 périodes et à grande vitesse que l'on rencontre fréquemment dans les stalions hydro-électriques. Les trois raisons essentielles de cette différence sont :

$1^{\circ} \mathrm{La}$ vitesse lente ;

$2^{\circ}$ La fréquence relativement faible;

$3^{\circ}$ Le grand rendement désiré.

Nous n̈insisterons pas sur l'influence de ces trois facteurs, nous nous contenterons de spécifier que la vitesse périphérique n'est que de $41 \mathrm{~m}$. par seconde alors qu'elle alleint pour des vitesses de 200 à $600 \mathrm{t} / \mathrm{m}$ et pour des machines de fréquences igales à 50 ou 60 périodes des vitesses linéaires de $60 \mathrm{~m}$. et mème 75 mètres par seconde.

Le fait que ces groupes sont les plus puissants construits jusqu'à ce jour serait une raison suffisante pour les décrire, mais à cette particularité s'ajoutent encore des dispositions

La figure 4 montre également que la silhonette de ces deux grcsses unités est analogue à celle des groupes de $32.500 \mathrm{KVA}$ installés depuis trois ans dans la même centrale. Celles-ci possèdent les caractéristiques suivantes :

$32.500 \mathrm{KVA}$ sous $\cos p=0,8-12.000$ volts, 150 tours minute 25 périodes.

Diamètre extérieur de la carcasse...... $6 \mathrm{~m} .550$

Hauteur de la carcasse............ 2 m. 870

Diamètre de l'arbre près de la bride d'accouplement ................

Diamètre de l'arbre à la bride d'accou-

plement....................

Charge totale sur le palier de butée....

Poids du rotor de l'alternateur .........

Charge due à la turbine et à la poussée de

l'eau ........................

$635 \mathrm{~mm}$.

$1.140 \mathrm{~mm}$.

217 tonnes

149 -

$68-$

Comparaison entre les alternateurs de 3.750 KWA installés à l'origine de la Centrale

et les deux dernières unités de $65.000 \mathrm{KVA}$.

Les deux unités de $65.000 \mathrm{KVA}$ ont été prévues pour débiter des courants triphasés au lieu de courants biphasés des onze premières machines. La tension est de 12.000 volts au lieu de 2.200 ; le type à inducteur tournant à l'intérieur de l'induit fixe a été adopté au lieu du type "ombrelle " à inducteur tournant à l'extérieur de l'induit fixe; le poids total du rotor de l'alternateur et de la turbine est supporté par un palier de butée placé à la partie supérieure du groupe tandis que les précédents avaient leur partie tournante supportée par un palier à huile sous pression placé à la partie inférieure du groupe; la turbine est située immédiatement en dessous de l'alternateur et forme un ensemble d'un encombrement réduit au minimum tandis que les anciens alternateurs étaient placés à $4 \mathrm{~m} .60$ au-dessus spéciales adoptées dans leur construction. La vue d'ensemble fait nettement ressortir la simplicité du montage. Le stator repose sur un bâti circulaire supportant lui-mème le palier de butée prévu pour la charge totale de la partie tournante dn

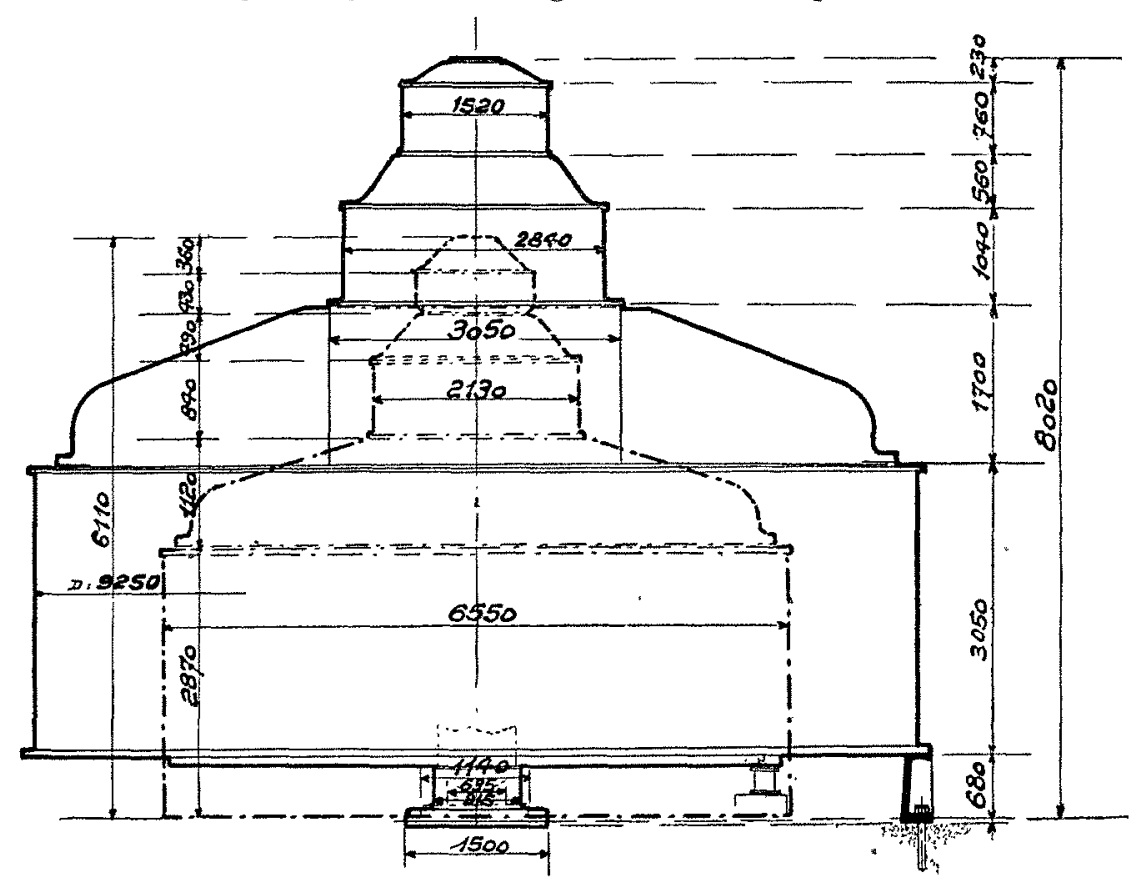

Fig. 4 groupe y compris la poussée de l'cau. Le palier guicle supérieur' fait corps avec le croisillon du palier de`butée. Le palier guide inférieur est placé immédiatement en dessous de la turbine; la proximité de l'alternateur et de la turbine permet d'éviter le palier guide intermédiaire que l'on rencontre généralement dans les installations similaires de puissance plus faible et que l'on place directement sous le rotor de l'alternateur. On remarque également l'alternateur monté en porte-à-faux à la partic supérieure du rotor principal. Ce petit générateur fournit le courant 


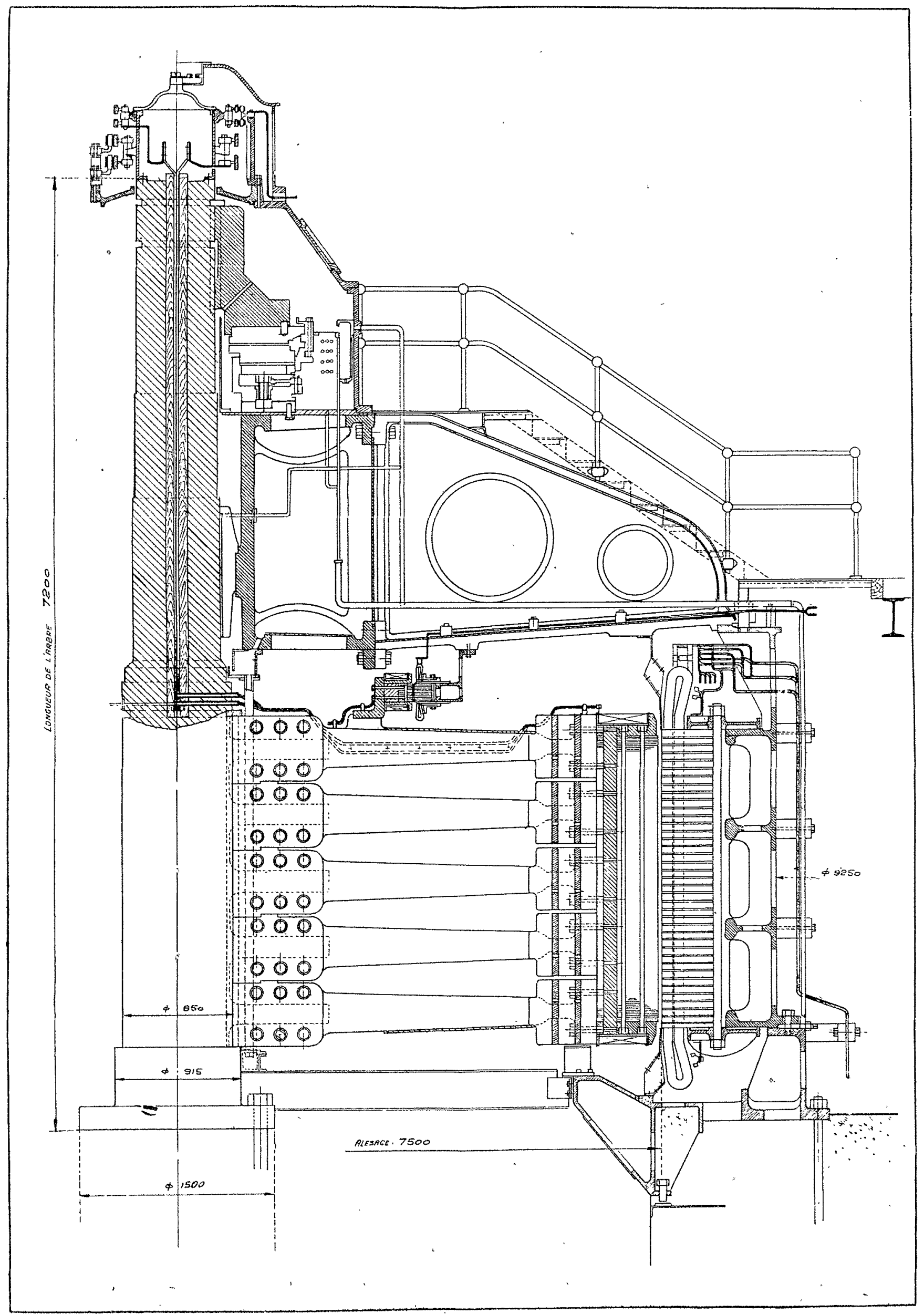

Fig. 5 
au groupe d'excitation ainsi qu'à tous les groupes auxiliaires de la centrale. Les bagues pour son excitation, ainsi que celles destinées à l'unité principale, sont montées sur l'arbre au-dessus du palier de butée. Le limiteur de vitesse prévu est monté à l'extrémité de l'arbre. Bagues et limiteur de vitesse sont protégés par des capots en fonte.

Stator. - La carcasse en fonte du stator est composée de 4 parties qui sont assemblées et dont chaque section pèse approximativement $19.500 \mathrm{~kg}$. (1), soit 78 tonnes pour la carcasse. Cette division est nécessaire pour des raisons de fonderie, d'usinage, de manutention à l'usine, de transport et de montage à la Station centrale. Le diamètre extérieur du stator est de 9.250 et sa hauteur de 3 mètres (2).

Les tòles au silicium du stator, à pertes très réduites, sont fixées par des clavettes aux traverses axiales de la carcasse et sont serrées entre deux flasques en acier moulé par 120 boulons en acier de $64 \mathrm{~m} / \mathrm{m}$ de diamètre, qui traversant de haut en bas le stator (entre la carcasse et les tôles) en augmentent la rigidité dans une notable proportion. Etant donné le grand poids de la machine, les tôles sont assemblées sur place lors du montage à la Station centrale. A ce sujet, il a été prévu un dispositif spécial pour le serrage par paquet d'un empilage réduit de façon à obtenir une grande régularité dans la totalité, constituée par 39 paquets séparés les uns des autres par des intervalles de ventilation de $12 \mathrm{~m} / \mathrm{m}$ environ de largeur. Le serrage énergique permet d'éviter les vibrations aux joints des segments, celles-ci étant le plus souvent la cause de bruits anormaux.

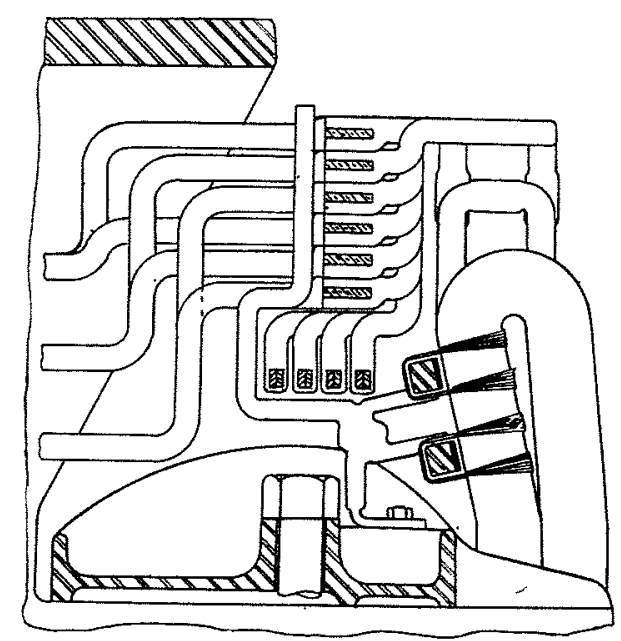

Fig. 6

La carcasse du stator est supportée par un socle en fonte pesant $24.500 \mathrm{kgs}$. Ce socle est partiellement encastré dans le massif de béton de la Station. 24 bagues de centrage et 24 boulons de $64 \mathrm{~m} / \mathrm{m}$ de diamètre servent à centrer la carcasse et à la fixer sur le socle. Il est prévu en plus 24 goujons disposés radialement, pour empècher tout déplacement du stator sur le socle en cas de court-circuit.

Bobinage induit. - Les bobines d'induit de cette machine sont au nombre de 360 et ont chacune $640 \mathrm{~m} / \mathrm{m}$ de largeur et $3 \mathrm{~m} .250$ de longueur. Chaque bobine isolée pèse environ $85 \mathrm{kgs}$.,

(1) L'alternateur à axe vertical de $9.000 K V A, K E O K L K$ Station de la Missipipi River Power $C^{\circ}, 11.000$ volts, 57,7 t/m, 25 périodes a un diametre extérieur de carcasse de $9 \mathrm{~m}$. 500 et le poids de la carcasse seule atteint 73 tonnes.

(2) L'alternateur à axe vertical de $10.000 K \mathrm{KA}$ sous $\operatorname{Cos} \varphi=0,75$ de la Cedars Rapids Manufacturing and Power Co, Québec, 6.600 volts, 55,6 t/m, 136 pôles, a une carcasse en quatre parties, d'un diamètre extérieur de $11 \mathrm{~m}$. 500, mais une hauteur de carcasse de 0 m. 920 .

l'isolement en est fait au mica. Elles sọt imprégnées de façon à éviter toute poche d'air ou autres gaz, tout en leur laissant une flexibilité suffisante pour faciliter leur entrée dans les encoches. Chacune d'elles comprend trois sections et chaque section est composee de 36 conducteurs. Les tètes de bobines sont fixées à des anneaux rendus solidaires des flasques latérales, de façon à éviter route déformation en cas de court-circuit. Les connexions sont faites à la partie supérieure de la machine.

La fig. 7 représentant la coupe d'une encoche du stator domne une idée exacte de la composition des conducteurs induits et de lépaisseur relative de l'isolement par rapport aux lóles.

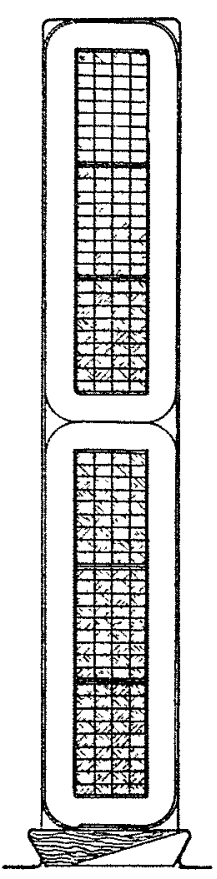

Fig. 7
Palier supérieur. - Le palier supéricur consiste en un moyeu en acier moulé de trois mètres de diamètre et $1 \mathrm{~m} .840$ de hauteur, pesant 19 tonnes. A ce moyeu viement se raccorder dix bras en acier moulé, chacun d'eux pesant $3.600 \mathrm{kgs}$. Le palier supérieur supporte la charge tolale : turbine, rotor de l'alternateur principal, rotor de l'alternateur d'excitation et poussée de l'eau dans la lurbine, estimée à 540 tonnes (1). Le palier et ses boulons de fixation sont isolés de la carcasse du stator pour éviter les courants de circulation se fermant par l'arbre qui pourraient amener des échauffements anormaux el détériorer les coussinets. Une plate-forme supportée par le moyeu central permet l'inspection de la bulé Kingsbury prévue avec refroidissement de l'huile par circulation d'eau. Les intervalles restés libres entre les bras du palier sont fermés par des tôles afin de diriger l'air servant à la ventilation et portent des ouvertures permettsnt la visite des bobinages.

La flèche calculée du palier était de $14 / 10 \mathrm{~mm}$ 4 , la flèche mesurée de $16 / 10 \mathrm{~mm}$.

Rotor. - En raison des grandes dimensions du rotor, la culasse inductrice est composée de cinq roues en acier moulé; chaque roue étant fondue en deux parties. Les deux moitiés sont boulonnées entre elles au moyen de 12 boulons en acier ou nickel de $90 \mathrm{~m} / \mathrm{m}$ de diamètre. Les jantes sont assemblées également par des boulons en acier au nickel. Les dix sections ont un poids total de 172 tonnes. Sur chaque demi-roue quatre éprouvettes d'essai sont prélevées de façon à pouvoir vérifier si les caractéristiques magnétiques et mécaniques imposées par le constructeur ont été respectées. Les cinq roues sont centrées les unes sur les autres par leur moyeu et clavetées sur l'arbre au moyen de deux clavettes diamétralement opposées. Elles laissent entre elles un intervalle entre leur jante ol assurent ainsi une ventilation suffisante pour refroidir les bobines inductrices. En raison de leurs grandes dimensions et de leur poids élevé, il n'a pas été possible de faire l'essai de l'ensemble du rotor en usine, mais chaque roue a cependant subi un essai de survilesse de $100 \%$ pendant deux minutes; le rotor devant être prévu pour supporter cet emballement de deux fois sa vitesse normale.

(1) Les allernaleurs de Queenston Chippawa (Canadal $45.000 \mathrm{KVA}$ sous Cos $\varphi=0.8$ triphasé 12.000 volts, $187 \mathrm{t} / \mathrm{m}, 25$ périodes ont un palier supérieur prévu pour supporter normalement près de 500 tonnes.

Les alternateurs triphasés à axe vertical de $40.000 \mathrm{KVA}$ sous $\operatorname{Cos} p=0,75$ de la Shawinigan Water and Power $C^{\circ}$, Québec (Canada), 11.000 volts, $138,5 \mathrm{t} / \mathrm{m}, 60$ périodes, mis en service en octobre 1922 , ont un palier de butée prévu pour 360 tonnes de charge maxima. 
L'arbre de ce générateur a un diamètre de $860 \mathrm{~m} / \mathrm{m}$, sous le moyeu de la culasse inductrice et un dianètre de $1.500 \mathrm{~m} / \mathrm{m}$ à la bride d'accouplement, celui-ci est réalisé par 18 boulons de $100 \mathrm{~m} / \mathrm{m}$ de diamètre.

Tôles polaires. - Les pôles inducteurs sont composés de tôles de $3 \mathrm{~m} / \mathrm{m}$ d'épaisseur, serrées entre deux flasques en acier moulé par deux boulons en acier au nickel qui traversent le pôle de part en part, au lieu de rivels comme on le fait dans la plupart des cas. Deux clavettes en acier au nickel traversent également les pôles, et les boulons de fixation de ceux-ci à la jante du rotor, viemnent s'y fixer.

Bobinage inducteur. - L'excitation est réalisée sous une tension de 250 volts. Les dimensions du cuivre inducteur sont de $11 \mathrm{~mm} \times 67 \mathrm{~mm}$. Ces dimensions sont probablement les plus grandes, utilisées jusqu'à ce jour dans un bobinage inducteur. La largeur de chaque bobine est approximativement de $53 \mathrm{c} / \mathrm{m}$, sa hauteur $41 \mathrm{c} / \mathrm{m}$, sa longueur $2 \mathrm{~m}$. 54 . Les bobines sont calées dans leur partie médiane par des cales qui empêchent les spires de se déformer sous l'effet de la force centrifuge.

Freins. - Douze freins à air sont montés sur une couromne en fonte encastrée dans le béton de la salle des machines. Ces freins fonctionnent à une pression d'environ $9 \mathrm{kgs} / \mathrm{cm}^{2}$ et peuvent arrêter le rotor en cinq minutes. Ces freins sont en réalité une combinaison de freins et de vérins qui servent à supporter le poids total : rotor alternateur et roue de la turbine, en cas de démontage du groupe. Quand on utilise le dispositif pour soulever le rotor, on obtient la pression approximative de $100 \mathrm{~kg} / \mathrm{cm}^{2}$ par une pompe à main à haute pression (Watson-Stillman). Toutes les canalisations et le système freins-vérins doivent résister à cette pression supplémentaire. Une soupape de contrôle est disposée sur la table d'opérations du machiniste.

Circulation d'huile. - De mème chaque unité peut ètre directement branchée sur le système de graissage général de la Station ou sur la pompe à huile indépendante affectée à chacune d'elles, prévue avec un filtre Richardson-Phénix. Le moteur triphasé du groupe a une puissance de $1 / 4$ de cheval à une vitesse de $750 \mathrm{t} / \mathrm{m}$ et est alimenté sous 220 volts $-25 \mathrm{p}$. p. s. La pompe doit fournir la quantité d'huile nécessaire pour le palier de guidage ainsi qu'une partie pour le palier de butée. Le palier de butée est refroidi par une circulation d'eau dans un serpentin en cuivre. Il faut environ 230 litres d'eau par minute pour le refroidissement précité. Des thermomètres indiquent la température de l'huile du palier de butée ainsi que du palier de guidage.

Ventilation. - L'air nécessaire à la ventilation du générateur est pris de chaque côté de la machine, en égale quantité. Il faut environ $60 \mathrm{~m}^{3}$ d'air par seconde pour la ventilation. A cet effet un ventilateur spécial est prévu.

Générateur d'excitation. - Ainsi qu'il avait été prévu déjà pour les grosses unités de l'Ontario Water Power Co, Canadian Niagara Falls et sur la proposition de Mr. Johnson, ingénieur de la N. F. P., l'excitation est fournie par un groupe indépendant.
Ce groupe, ainsi que tous les auxiliaires de la Centrale, sont alimentés par l'alternateur monté en porte à faux sur la roue supérieure du rotor de l'alternateur principal. Sa puissance est de $650 \mathrm{KVA}$, soit exactement $1 \%$ de la puissance du générateur, il fournit du courant triphasé $2.200 \mathrm{~V}$. à 25 p. p. s. et sa surélévation de température garantie à $\operatorname{Cos} \subsetneq=0,8$ est de $50^{\circ} \mathrm{C}$, mesurée au thermomètre pour le stator, et de $55^{\circ} \mathrm{C}$ : surélévation mesurée par détecteurs placés au nombre de 6 dans le bobinage. La température garantie du rotor est de $55^{\circ} \mathrm{C}$ mesurée par résistances. Les bobines induites ont un isolement prévu très large pour ce voltage relativement réduit.

Ce générateur a été construit de telle sorte que les pôles peuvent être passés entre les bras du palier supérieur du groupe. Avec cette disposition il est possible de remplacer les bobines induites de l'alternateur d'excitation en démontant un nombre suffisant de ses pôles, et sans avoir à toucher aucune partie de l'alternateur principal, sauf évidemment les tôles d'obturation qui se trouvent entre les bras du palier supéricur.

Dimensions el poids. - Le diamètre extérieur du socle est de $9 \mathrm{~m}$. 5 et la hauteur totale de l'alternateur au-dessus du sol de la salle des machines est de 8 mètres; le poids atteint $680 \mathrm{~T}$.

Il est intéressant de noter les dimensions suivantes, qui découlent de la description faite dans l'article de M. G. Foster, ingénieur de la G. E. Co (A. I. E. E., avril 1924).

\section{Dimensions.}

Hauteur de la carcasse.................. $3 \mathrm{~m}$. 050

Empilage tòles stator..................... 2 m. 300

Alésage $\ldots \ldots \ldots \ldots \ldots \ldots \ldots \ldots \ldots \ldots \ldots, 7 \mathrm{~m} .500$

Diamètre extérieur des tôles stator........... $8 \mathrm{~m} .250$

Hauteur du pôle....................... 0 m. 500

Diamètre de l'arbre sous le manchon inducteur.... $00 \mathrm{~m} .860$

Diamètre près du tourteau d'accouplement....... 0 m. 900

Diamètre à la bride d'accouplement avec la turbine. $1 \mathrm{~m} .500$

Longueur totale de l'arbre................. $7 \mathrm{~m} .200$

Vitesse linéaire ..................... $41 \mathrm{~m} . \sec 5$

Fixation des pôles inducteurs, par pôle, 20 boulons de $57 \mathrm{~mm}$. de diamètre.

\section{Poids.}

Carcasse, tôles, enroulements, flasques (stator).. Poids du cuivre induit (connexions comprises) ..

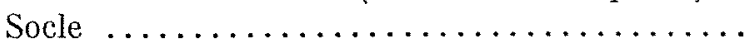

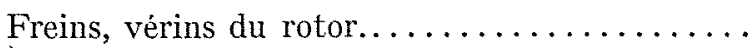

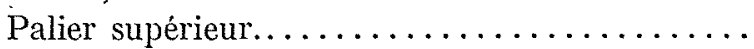
Rotor complet avec arbre................ L'arbre seul........................ Un pôle inducteur avec son bobinage .......... Poids du cuivre inducteur par pôle............. Divers ............................ Poids total du générateur principal........... Poids de l'excitatrice.................... $\mathrm{PD}^{2}=2.650$ tonnes $/ \mathrm{m}^{2}$.

(Journal A. I. E. E. - Avril 1924).
215 tonnes $32-$ 23 T. 800

$26 \mathrm{~T} .600$

54 tonnes $355-$

$27-$

15 tonnes

$680-$

$11-$
1.260 kilos 